\title{
Desenvolvimento biomecânico e avaliação de uma nova estrutura para reabilitação all-on-four.
}

\author{
Vanessa Araujo SILVA (1), Allyson Henrique de Andrade FONSECA (1), \\ Dimitri Ribas FONSECA (2) e Paulo Isaías SERAIDARIAN (1)
}

\begin{abstract}
ARTIGO ORIGINAL
Resumo

Introdução: Para a reabilitação completa de mandíbulas totalmente edêntulas, a opção de carga imediata determina o uso de próteses provisórias que imobilizam os implantes instalados. Embora as próteses provisórias com reforço proporcionem reabilitações com benefícios biomecânicos, a não adoção desta recomendação coexiste em diferentes estudos sobre restaurações imediatas.

Objetivo: Este estudo avalia um tipo de prótese para restaurar mandíbulas pelo conceito all-on-four. Material e método: O comportamento mecânico das próteses com a estrutura modificada foi avaliado in vitro, sob um ensaio de flexão mecânica unilateral em cantilever. Dois grupos representativos foram incluídos no conceito all-on-four, com um grupo teste $G 1(n=10)$ contendo estruturas modificadas e um grupo controle $\mathrm{G} 2(n=10)$ que incluiu próteses acrílicas completas. As amostras foram submetidas à termociclagem com 500 ciclos $\left(5^{\circ} \pm 2^{\circ} \mathrm{C}\right.$ por 30 se $55^{\circ} \pm 2^{\circ}$ por $\left.30 \mathrm{~s}\right) \mathrm{e}$ ao ensaio de flexão mecânica no cantilever.

Resultado: $O$ teste de Mann-Whitney revelou diferença significativa entre G1 e G2 ( $p<0,001)$. Na avaliação descritiva, o $\mathrm{G} 1$ apresentou média de $830,50 \mathrm{~N}$ até a fratura inicial da resina, enquanto o grupo controle apresentou média de $403,58 \mathrm{~N}$. A resistência máxima até a fratura completa foi registrada no G1, com média de $903,03 \mathrm{~N}$, enquanto no $\mathrm{G} 2$, foi registrada uma média de $435,20 \mathrm{~N}$. O componente linear vertical da flexão foi de $0,68 \mathrm{~mm}$ e $0,39 \mathrm{~mm}$ até a fratura inicial da barra, respectivamente para G1 e G2.
\end{abstract}

Conclusão: A estrutura modificada para o protocolo all-on-four determinou melhor desempenho mecânico quando comparado ao mesmo modelo de prótese acrílica completa.

Palavras Chave: Carga imediata no implante dentário; mandíbula; estrutura; implantes dentários.

Instituição afiliada: 1- PUC Minas - Pontifícia Universidade Católica de Minas Gerais, Departamento de Odontologia, Belo Horizonte, MG, Brasil. 2- UFVJM - Universidade Federal dos Vales do Jequitinhonha e Mucuri, Departamento de Odontologia, Diamantina, MG, Brasil.

Dados da publicação: Artigo recebido em 01 de agosto, revisado em 10 de agosto, aceito para publicação em 19 de agosto e publicado em 29 de Agosto.

DOI: https://doi.org/10.36557/2674-8169.2020v2n9p03-16

Vanessa Araujo Silva vanessa.araujos@icloud.com 


\section{Biomechanical development and evaluation of a new structure for all-on-four rehabilitation.}

Introduction: For the complete rehabilitation of fully edentulous mandibles, the option of immediate loading determines the use of temporary prostheses that immobilize the installed implants. Although temporary prostheses with reinforcement provide rehabilitation with biomechanical benefits, the failure to adopt this recommendation coexists in different studies on immediate restorations.

Objective: This study evaluates a type of prosthesis to restore jaws using the all-on-four concept.

Material and method: The mechanical behavior of the prostheses with the modified structure was evaluated in vitro, under a unilateral mechanical flexion test in cantilever. Two representative groups were included in the all-on-four concept, with a G1 test group $(n=10)$ containing modified structures and a $G 2$ control group $(n=10)$ that included complete acrylic prostheses. The samples were subjected to thermocycling with 500 cycles $\left(5^{\circ} \pm 2^{\circ} \mathrm{C}\right.$ for $30 \mathrm{~s}$ and $55^{\circ} \pm 2{ }^{\circ}$ for $30 \mathrm{~s}$ ) and the mechanical flexion test on the cantilever.

Result: The Mann-Whitney test revealed a significant difference between G1 and G2 ( $p$ $<0.001$ ). In the descriptive evaluation, G1 showed an average of $830.50 \mathrm{~N}$ until the initial fracture of the resin, while the control group had an average of $403.58 \mathrm{~N}$. Maximum resistance to complete fracture was recorded in $\mathrm{G} 1$, with an average of $903.03 \mathrm{~N}$, while in $\mathrm{G} 2$, an average of $435.20 \mathrm{~N}$ was recorded. The vertical linear component of flexion was $0.68 \mathrm{~mm}$ and $0.39 \mathrm{~mm}$ until the initial bar fracture, respectively for $\mathrm{G} 1$ and $\mathrm{G} 2$.

Conclusion: The modified structure for the all-on-four protocol determined better mechanical performance when compared to the same model of complete acrylic prosthesis.

Descriptors: Immediate loading on the dental implant; jaw; structure; dental implants..

Instituição afiliada: 1- PUC Minas - Pontifícia Universidade Católica de Minas Gerais, Departamento de Odontologia, Belo Horizonte, MG, Brasil. 2- UFVJM - Universidade Federal dos Vales do Jequitinhonha e Mucuri, Departamento de Odontologia, Diamantina, MG, Brasil.

Dados da publicação: Artigo recebido em 01 de agosto, revisado em 10 de agosto, aceito para publicação em 19 de agosto e publicado em 29 de Agosto.

DOI: https://doi.org/10.36557/2674-8169.2020v2n9p03-16 Vanessa Araujo Silva vanessa araujos@icloud.com 


\section{INTRODUÇÃO}

O conceito all-on-four (Nobel Biocare, Gotemburgo, Suécia), uma modalidade de reabilitação que combina implantes axiais anteriores e angulares posteriores com carga imediata na mandíbula, foi validado por estudos longitudinais ${ }^{1,2}$. Uma vez que foi desenvolvido por Maló et al. $^{3}$, a técnica cirúrgica-protética tem mostrado excelentes resultados e taxas de sucesso previsíveis ${ }^{4-6}$.

Após o acompanhamento de 5 e 10 anos, foram observadas taxas de sucesso do implante de $98,1 \%$ e $94,8 \%$, respectivamente. Excelentes resultados também foram obtidos nas avaliações biomecânicas da reabilitação protética da mandíbula edêntula, por meio do conceito all-on-four ${ }^{2}$. Com a utilização de quatro implantes, balanceados por maior espaçamento entre eles, a configuração poligonal esperada é mantida, garantindo estabilidade biomecânica suficiente à prótese e distribuição de força adequada ao osso 5, 7-9 .

As vantagens desse conceito incluem a possibilidade de utilização de implantes longos e a redução da necessidade de enxerto ósseo ${ }^{8}$. A prevenção de longos cantiléveres, bem como a possibilidade de aumentar a distância entre os pilares anterior e posterior, caracterizam o significado da proposta ${ }^{9,10}$. Outras vantagens incluem menor custo e facilidade de limpeza, devido ao reduzido número de implantes ${ }^{5,11}$.

No entanto, diversos estudos relatam complicações protéticas e quebras temporárias de próteses acrílicas durante a fase de recuperação pós-operatória ${ }^{4,5,9,10}$. A escolha do preparo da prótese provisória sem reforço interno contribui para a ocorrência de complicações que variam entre 10,8\%, segundo Hinze et al. ${ }^{5}$ e $30 \%$ relataram Maló et al. $\underline{3}$ estudo, enquanto as próteses provisórias, com reforço interno, são consideradas padrão ouro por oferecerem vantagens biomecânicas ${ }^{11}$. A estrutura metálica promove imobilização rígida e mantém os micromovimentos do implante abaixo do limite crítico, favorecendo a osseointegração ${ }^{12}$. O reforço metálico da prótese dificulta a fratura e também pode reduzir as forças de flexão, diminuindo o estresse ósseo e favorecendo a dureza necessária durante a fase de cicatrização do implante ${ }^{8}$.

No entanto, um reforço metálico de qualidade, garantindo um ajuste passivo da prótese, requer mais tempo e aumenta os custos financeiros da reabilitação ${ }^{1}$. Alternativas para o planejamento de frameworks eficazes têm sido discutidas em diversos estudos $\underline{8}, \underline{12}-\underline{15}$. Apesar dos avanços, não foram observadas evidências $\underline{4}, \underline{6}, \underline{8}, \underline{11}$ que complementassem a simplicidade proposta por Maló et al. $\underline{2}, \underline{3}$.

O presente estudo propõe um novo tipo de prótese imediata para o protocolo all-onfour, constituída por estrutura metálica não soldada, projetada para oferecer uma visão in vitro do comportamento mecânico dessas próteses quanto à sua resistência à fratura, quando comparada ao acrílico totalmente próteses sem reforço interno. 


\section{MATERIAL E MÉTODO}

Dois conjuntos de corpos de prova foram comparados, um grupo de teste denominado G1 ( $n=10)$ e o outro, $G 2(n=10)$. O G1 incluiu próteses com estrutura metálica em liga de cobalto-cromo não soldada, fundidas e usinadas separadamente do cilindro suporte e posteriormente combinadas com cilindros de titânio usinados e pré-fabricados durante a prensa de acrílico. ${ }^{\circ} \mathrm{G} 2$ consistiu em próteses totalmente acrílicas para o protocolo all-onfour.

O enceramento prévio e a montagem dos dentes de acrílico foram realizados para reproduzir a distribuição dentária ideal, em prótese mandibular completa com protocolo Brånemark, permitindo o ajuste da geometria e posicionamento do implante. A partir desse enceramento, foi desenvolvido um modelo diagnóstico, em resina acrílica termoativada, contendo quatro análogos de minipilares cônicos com plataforma de 4,1 mm, banda de $3 \mathrm{~mm}$ e convergência de 20 graus (Neodent, Curitiba, PR, Brasil ) Os dois análogos anteriores foram posicionados perpendicularmente às áreas correspondentes ao cíngulo dos incisivos laterais, e os dois análogos posteriores foram posicionados na região do segundo pré-molar, conforme Maló et al. ${ }^{3}$. Para simular o quadro clínico pós-cirúrgico imediato, após a inserção dos minipilares protéticos, os análogos posteriores do modelo diagnóstico foram inseridos sem inclinação para a plataforma protética paralela ao plano oclusal para representar o ângulo distal do implante (30 graus).

Por meio de um índice de transferência (Figura 1a), com componentes de moldagem constituídos por hastes metálicas conectadas por acrílico de baixa contração, a posição dos componentes protéticos foi transferida para um disco metálico de aço carbono, constituindo o modelo metálico mestre (MM) ( Figura 1 b). O MM foi preparado para ser acoplado também à máquina de ensaio, funcionando como suporte para o ensaio mecânico. Com o auxílio do software AutoCAD 2007 (Autodesk, Califórnia, EUA), foi preparada uma estrutura metálica para reforço protético compatível com o protocolo all-on-four ( Figura 1c), adotando as seguintes dimensões: largura gengival-oclusal de 2,0 mm, largura vestíbulolingual de 6,8 $\mathrm{mm}$ e cantilever de $10 \mathrm{~mm}$. Quatro brocas circulares de 4,9 $\mathrm{mm}$ de diâmetro foram projetadas para inserir os cilindros de suporte (Neodent, Curitiba, PR, Brasil). Com este diâmetro, a estrutura percorreu o formato cônico dos cilindros, com encaixe cervical no ombro e suporte de $0,6 \mathrm{~mm}$ em toda a abertura. Após a finalização do layout, o framework foi impresso em 3D com o suporte de uma impressora SD 300 Pro (SKA, Tel Aviv, Israel). 


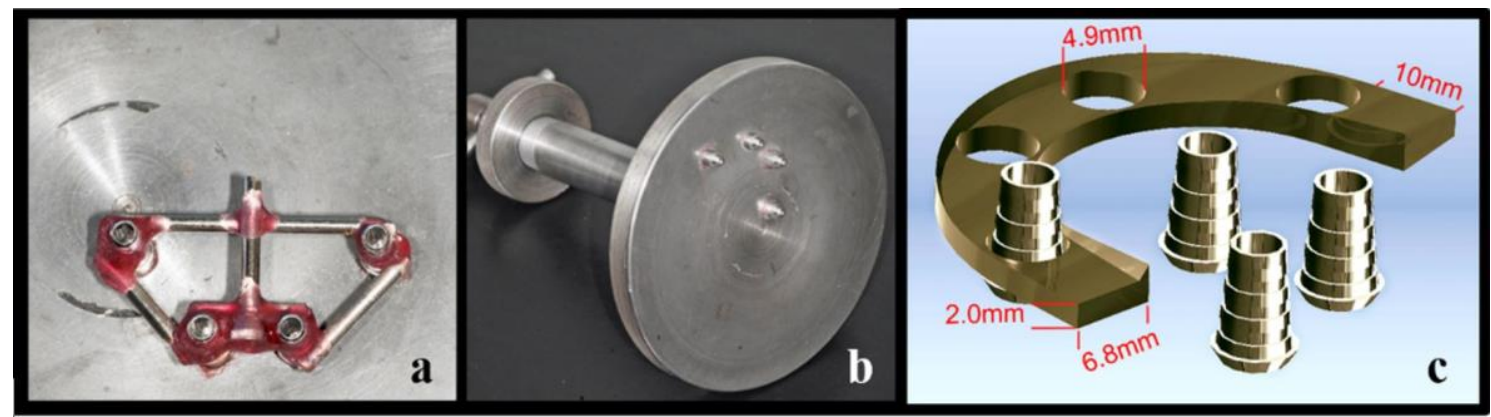

Figura 1 (a) Imagem de índice de transferência; (b) Modelo mestre (MM); (c) modelo de projeto CAD para a barra de metal.

A partir do protótipo, foi possível desenvolver uma matriz metálica (M0) projetada para reproduzir os padrões de resina sob prensagem (Pattern Resin LS GC, Tóquio, Japão) para as futuras estruturas metálicas ( Figura 2 a). Após a verificação das dimensões com auxílio de paquímetro, foram selecionadas 20 estruturas de acrílico para constituir os dois grupos experimentais. Para o G1, dez estruturas foram processadas e fundidas em liga de cobaltocromo (Co-Cr) ( Figura 2b). Para o G2, foram reservados dez padrões de acrílico, livres de qualquer processamento.

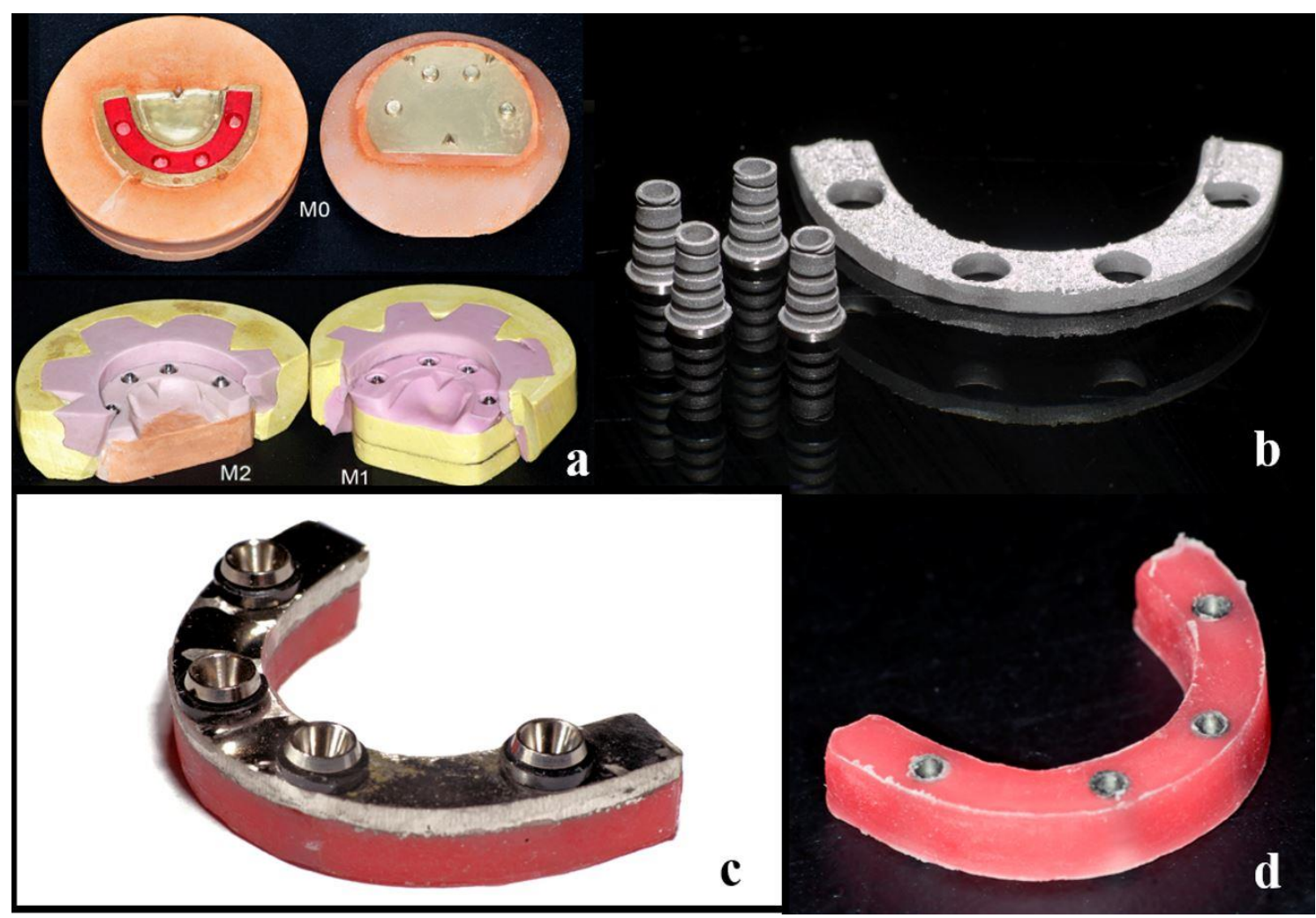

Figura 2 (a) Matrizes; (b) barras de metal não soldadas; (c) corpo de prova G1 em cera; (d) Amostra de teste $\mathrm{G} 2$ em cera.

A seção da prótese destinada a prensar a resina foi reproduzida sobre duas outras matrizes (M1 e M2) ( Figura 2 a). O M1 possibilitou o posicionamento das estruturas do G1 e o M2 foi preparado para o controle do G2. O M1 foi diferente por espaçar a estrutura a 1,5 
$\mathrm{mm}$ do ombro do cilindro, enquanto no $\mathrm{M} 2$ as estruturas entraram em contato entre si. Ambas as matrizes têm análogos de implante para serem aparafusados aos cilindros quando as próteses são montadas.

Ambas as matrizes permitiram a montagem dos protótipos em cera, com largura vestíbulo-lingual de $6,8 \mathrm{~mm}$ e altura cérvico-oclusal de $6,9 \mathrm{~mm}$, incluindo armações e cilindros de titânio ( Figuras 2 e 2d). Em M1, foram inseridos anéis de borracha (1,5 mm) sobre o ombro do cilindro de titânio para garantir homogeneidade entre o espaçamento e as barras ( Figura 2c).

Após verificados com paquímetro, os protótipos foram aparafusados em vinte modelos, constituindo cópias do modelo MM, com análogos de titânio e bases em gesso tipo IV (Herostone, Vigodente, RJ, Brasil). Em conjuntos de quatro unidades, os modelos com seus respectivos protótipos foram incluídos em mufla. Após um tempo de prensa de gesso de aproximadamente 30 minutos, a mufla foi levada ao forno de micro-ondas (LG 1200W, LG Electronics, SP, Brasil) por um minuto e vinte segundos para derreter a cera. Após a remoção total da cera, os cilindros pré-fabricados foram limpos com pincel e jateados com partículas de óxido de alumínio de 125 micrômetros, e novamente aparafusados aos modelos incluídos nas mufla. Frameworks G1também foram jateados com óxido de alumínio. Pó e líquido de resina ativada por micro-ondas (VipWave, Vip, São Paulo, SP, Brasil) foram dosados de acordo com as instruções do fabricante e depositados dentro da mufla, que foi prensada a 1 ton por 20 minutos. Seguindo as instruções do fabricante para polimerização acrílica total, na etapa inicial a mufla foi mantida por 20 minutos a $10 \%$ a $20 \%$ da potência em forno de micro-ondas, e na etapa final, por 5 minutos a $30 \%$ a $40 \%$ da potência .

O espaçamento entre a barra metálica do cilindro de suporte e o ressalto no grupo G1, após a polimerização, possibilitou a formação de um colar de resina que ligava essas estruturas. Após desembaraçar, os corpos de prova foram limpos com uma escova e armazenados em água a $37^{\circ} \pm 1^{\circ} \mathrm{C}$ até a ciclagem térmica.

Os corpos-de-prova foram aparafusados em modelo diferente, também originado do MM, e grupos de dez unidades foram submetidos a 500 ciclos em termociclador (MSCT / 3eELQUIP, São Paulo, SP, Brasil). Cada ciclo consistiu em banhos de 30 segundos, alternados em água a $5^{\circ} \pm 2^{\circ} \mathrm{C}$ e $55^{\circ} \pm 2^{\circ} \mathrm{C}$. Terminada a termociclagem, os corpos de prova foram avaliados visualmente por meio de lupa estereoscópica, STEMI 2000-C (Zeiss, Alemanha), com aumento de 20x, por examinador devidamente calibrado, que não observou danos aparentes nos corpos de prova. Após a ciclagem térmica, os corpos de prova foram armazenados novamente em água a $37^{\circ} \pm 1^{\circ} \mathrm{C}$.

O MM foi acoplado a um testador universal (EZTest 5kN, EZ-L, Shimadzu, Japão); aqui, os corpos de prova $\mathrm{G} 1$ e $\mathrm{G} 2$ foram aparafusados individualmente com torque de $20 \mathrm{~N}$ em cada parafuso de titânio. Um pistão de carregamento foi posicionado sobre o cantilever direito, distante $5 \mathrm{~mm}$ da borda distal da barra, exercendo uma força axial de compressão com limite 
máximo de $1.000 \mathrm{~N}$. A velocidade aplicada foi de $0,5 \mathrm{~mm} /$ minuto até a fratura do corpo de prova ou até atingir a carga máxima ( Figura 3 ).

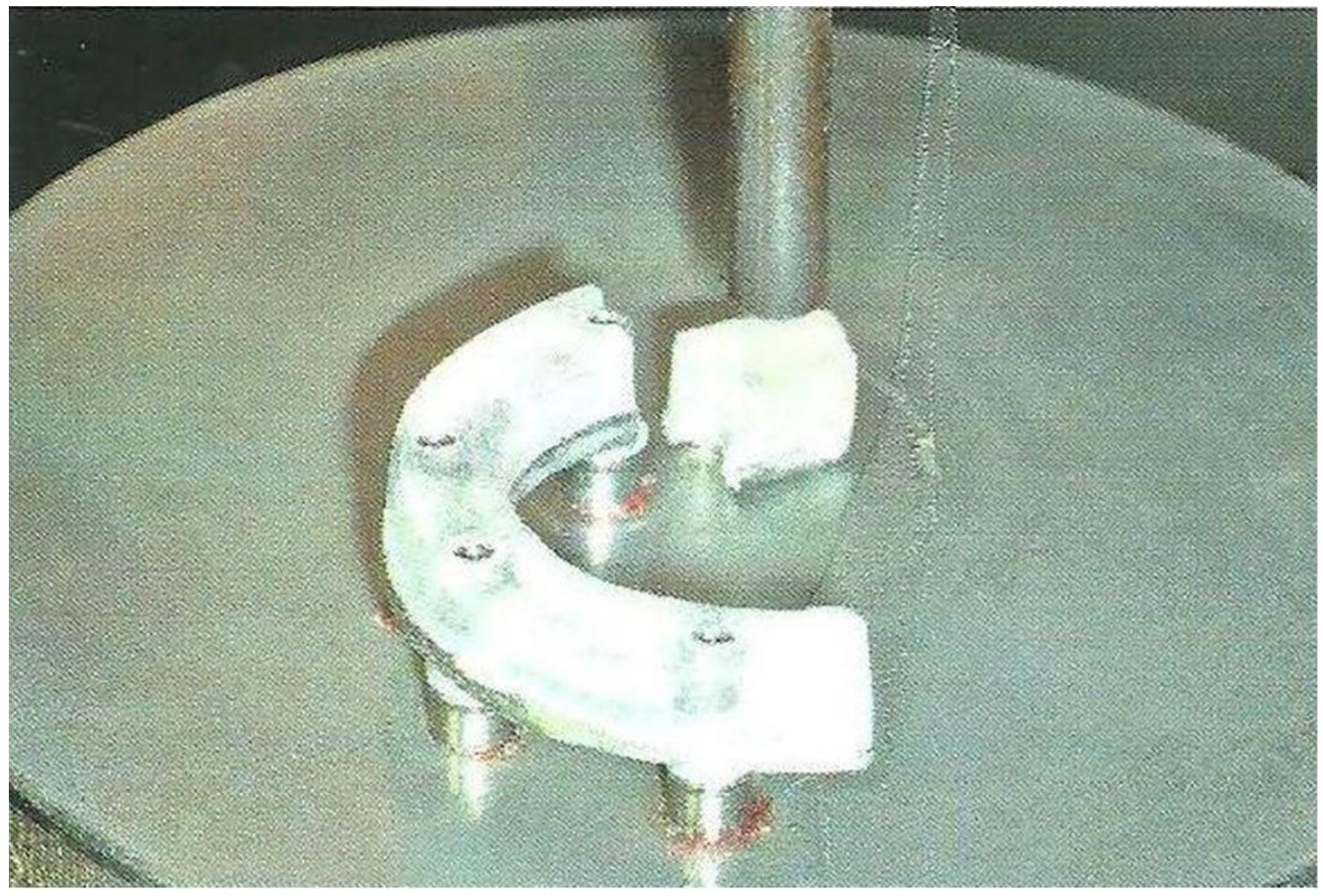

Figura 3 Amostra de teste G1 após a fratura.

Este estudo é uma adaptação para o português do artigo original "SILVA, Vanessa Araujo, et al. "Biomechanical development and evaluation of a new framework for all-on-four rehabilitation." Revista de Odontologia da UNESP 48 (2019). "27" E segue os preceitos Creative Commons - Atribuição 4.0 Internacional - CC BY 4.0 de livre distribuição, uso ou remixagem. Nenhum dos autores endossou este compartilhamento

\section{RESULTADO}

A ciclagem térmica, avaliada com lupa, não produziu danos aparentes nos corpos de prova. O teste de flexão ocasionou fraturas completas nos cantiléveres do corpo de prova que se propagam pela interface vestíbulo-lingual, adjacente ao cilindro distal, em um corte transversal da barra ( Figura 3 ). As fraturas iniciais (FI) ocorreram na resina, que, à medida que o teste avançava, tornou-se cheia de fraturas (FC), caracterizadas pela queda repentina de tensão registrada pelo software da máquina de ensaio.

A Figura 4 apresenta os gráficos de deformação (\%) e carga aplicada (N) gerada no Testador Universal, com evidências de fratura inicial (FI) e fratura completa (CF). A Tabela 1 apresenta a análise descritiva das variáveis dependentes. O teste de Mann Whitney revelou significância estatística na comparação intergrupos para a carga suportada até a fratura inicial 
da resina $(p<0,001)$, carga máxima aplicada $(p<0,001)$, bem como no componente linear vertical da flexão do cantilever $(p<0,001)$.

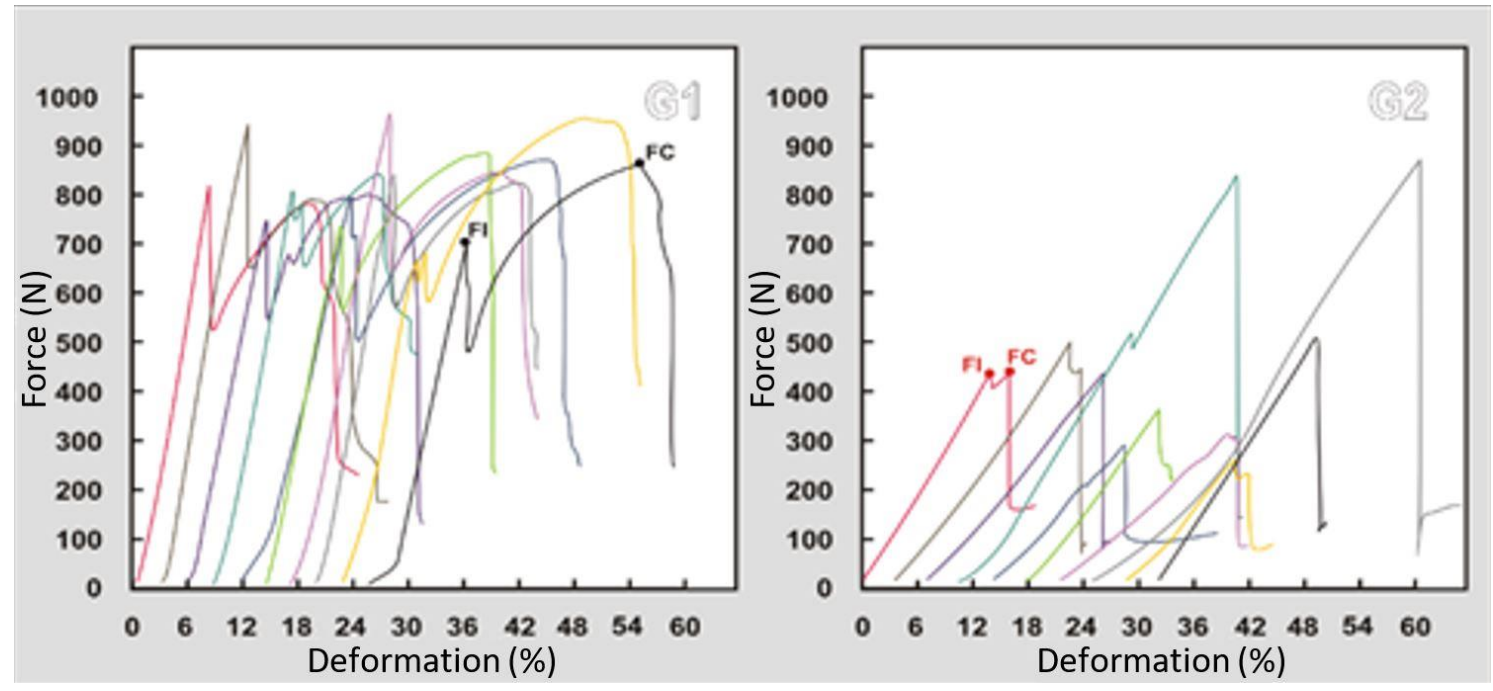

Figura 4 Gráfico de deformação (\%) e carga aplicada (N), registrada para G1 e G2.

Tabela 1 Análise do comportamento mecânico G1 e G2 (n = 10)

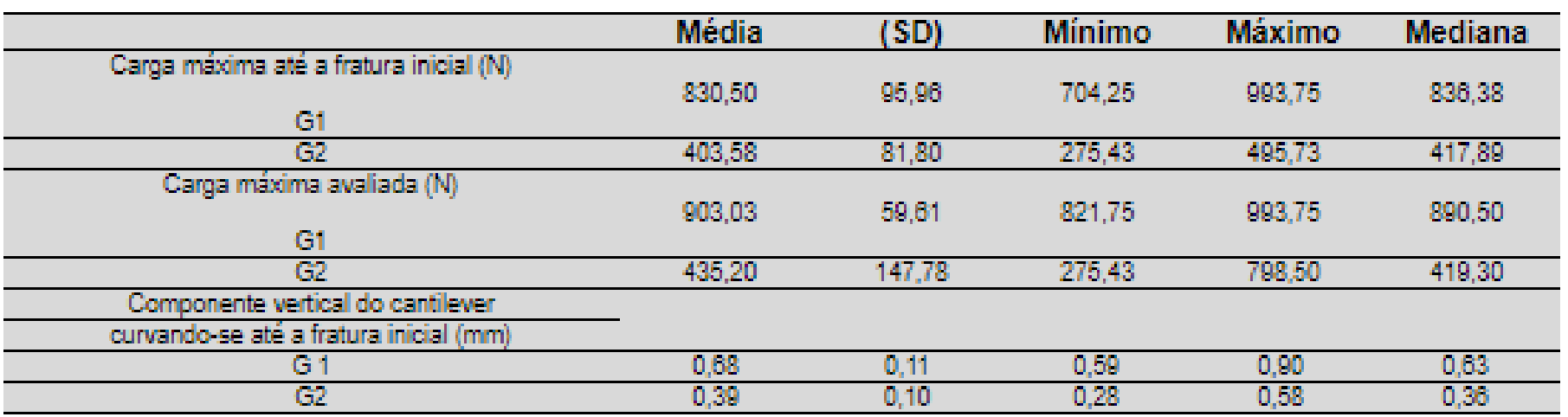

No G1 ocorreram fraturas simultâneas de resina e metal e todas as estruturas metálicas quebraram com cargas mínimas acima de $821 \mathrm{~N}$ e cargas máximas de $993,75 \mathrm{~N}$. No G2, todas as barras quebraram com carga mínima de $275,43 \mathrm{~N}$ e carga máxima de $798,50 \mathrm{~N}$.

\section{DISCUSSÃO}

O sucesso do conceito all-on-four foi clinicamente revisado para a taxa de sobrevivência dos implantes ${ }^{16}$, apesar da ocorrência de complicações protéticas, variando de $10,8 \%$ a $30 \%$ no total próteses provisórias, para reabilitação de mandíbulas edêntulas ${ }^{10}$. As maiores taxas de ocorrência foram encontradas em trabalhos que não utilizaram estrutura de reforço para as próteses ${ }^{3}$. Da mesma forma, no presente estudo, as diferenças de resistência à flexão entre G1, com estrutura metálica, e G2, sem tal armadura, foram significativas. 
A Figura 4 mostra que, no G1, houve maior absorção de carga, com menor percentual de deformação. No início da deformação, as linhas correspondentes a cada corpo de prova são perceptivelmente mais íngremes, quando comparadas com G2. O comportamento em G1 revela maior dureza, visto que a relação $\mathrm{N} / \mathrm{m}$ é maior quando comparada a G2 ${ }^{17}$.

Embora Maló et al. ${ }^{3}$ relataram que as falhas protéticas registradas eram reparáveis e não afetavam as taxas de sobrevivência. Este estudo detectou fraturas completas na extremidade do cantilever. Quando ocorrem clinicamente, as fraturas completas requerem desamarramento da prótese para reparo, no período de cicatrização entre 4 e 6 semanas, quando operadas com carga imediata ${ }^{18}$. A remoção precoce pode levar a um contra-torque excedendo a resistência do osso a ser remodelado e pode interferir na osseointegração ${ }^{12}$. Portanto, a estrutura metálica analisada neste estudo, pode contribuir para a eliminação de futuros problemas protéticos.

Vários estudos $1,5,9,10$ não fazem referência ao uso de estrutura metálica, nem discutem as evidências obtidas com a exclusão da armadura. A decisão tomada pode ter sido influenciada pela dificuldade em desenvolver, no período pós-cirúrgico imediato, uma estrutura de qualidade e baixo custo que permita resistência e adaptação passiva.

A resistência à flexão é conhecida por avaliar a flexão máxima que o material pode suportar antes da fratura ${ }^{19}$. Tendo em vista a ocorrência de extensão distal livre, submetida à função mastigatória, a resistência à flexão torna-se um fator clínico relevante nas reabilitações all-on-four.

A proposta de um projeto que proporcionasse espaçamento entre a barra metálica e o ombro do cilindro suporte, permitindo, após a prensagem, a formação de um colar de resina que unisse essas estruturas, buscou maior absorção de carga oclusal e melhor dissipação entre os implantes, evitando contato direto entre metais. Estudos que avaliaram as concentrações de estresse em próteses totais na mandíbula edêntula revelaram uma sobrecarga no implante distal $^{4,6}$. Mesmo quando foram selecionados implantes em ângulo distal , com possibilidade de redução da concentração de tensões de até $17 \%$, o acúmulo de tensões para implantes adjacentes em cantilever ainda persiste ${ }^{20}$.

O projeto da barra de metal com modificação estrutural permitiu que a montagem atingisse um componente linear-vertical de flexão de 0,68 $\mathrm{mm}$ até a fratura inicial dos corpos de prova G1. O aumento nesta extensão linear de flexão atingiu $74,36 \%$, considerado satisfatório quando comparado ao G2. Todas as fraturas, entretanto, ocorreram na interface distal do último implante, o que confirma a concentração de tensões apresentada nos achados de Begg et al. ${ }^{6}$ na biomecânica distal do implante.

Em projetos, incluindo uma barra isolada dos cilindros, exibindo relativa liberdade espacial entre eles e a estrutura, o elemento de resistência em observação neste estudo tornase a interface entre a resina, o cilindro e a barra de metal. Portanto, este estudo propôs a avaliação de todas as barras com resina submetidas à ciclagem térmica ${ }^{21}$, simulando a fadiga 
térmica da montagem. Mesmo com o envelhecimento térmico, os resultados da carga absorvida até a fratura inicial foram superiores aos do grupo controle e superiores à média observada em outros estudos ${ }^{4,6}$.

A prótese avaliada no G1 suportou a carga oclusal no cantilever $(830,50 \mathrm{~N})$ até a fratura inicial da resina. Levando-se em consideração que a carga oclusal registrada na região molar em pacientes com tratamento com implante apresentou uma variação entre $112,5 \mathrm{~N}$ e $450 \mathrm{~N}^{22-24}$, pode-se avaliar o incremento de resistência para a estrutura proposta neste estudo. Da mesma forma, o grupo controle G2 suportou a carga de flexão $(403,58 \mathrm{~N})$, valor próximo aos encontrados em trabalhos anteriores $1,5,9,10$ em relação à carga oclusal e pode explicar uma maior ocorrência de falha em próteses sem barras de reforço.

A barra permitia a exclusão das soldagens metálicas ${ }^{12,13}$, por serem fundidas em um único bloco. A fundição da estrutura, independente dos cilindros, contribuiu para a ausência de influência da contração de resfriamento da liga metálica e falhas de fundição inerentes ao processo de obtenção de estruturas convencionais em adaptação protética no presente estudo, considerando que a conexão aos cilindros ocorreu durante a prensagem Estágio. Nessa etapa, a colocação dos cilindros sobre o modelo mestre proporcionou melhor controle de sua posição.

No presente estudo, a cópia do modelo mestre (M1) foi a base para a prensagem das próteses. Os cilindros e a barra metálica foram fixados a este modelo, garantindo fidelidade ao MM após a prensagem. Portanto, para o uso clínico desta proposta, seria importante a obtenção de modelos fiéis da distribuição do implante na boca. A opção pela utilização do índice de transferência com barras metálicas auxiliares e baixa contração acrílica foi baseada em trabalhos que mostraram resultados satisfatórios com esta técnica ${ }^{25}$.

O grupo G2 apresentou fraturas iniciais com cargas inferiores às observadas no grupo G1. A menor resistência mecânica observada na barra totalmente acrílica comprova os achados do estudo clínico realizado por Maló et al. ${ }^{3}$ Apesar das taxas de fratura relatadas em diferentes estudos e do comportamento desse grupo controle, estruturas que não são totalmente acrílicas ainda são mencionadas em protocolos de restauração de mandíbulas desdentadas $5,9,10,26$.

São necessários apenas dois momentos clínicos pós-cirúrgicos para instalar a prótese provisória de acrílico, associada à barra não soldada, e para colocá-la em funcionamento. Além disso, a oportunidade de não utilizar soldas ou cimento para unir os cilindros à barra oferece resultados eficazes com baixo custo, apresentando simplicidade e rapidez. Devido às suas propriedades mecânicas, a prótese provisória de acrílico reforçada com metal pode ser utilizada por um período maior além da cicatriz, podendo também ser utilizada como prótese final de menor custo ${ }^{3,12}$. 
Este estudo apresentou limitações, principalmente pelo baixo número de amostras testadas e por se tratar de um estudo in vitro. Estudos longitudinais in vivo são garantidos para alcançar resultados clínicos que permitem uma comparação com este estudo in vitro .

\section{CONCLUSÃO}

Os resultados confirmaram que o reforço metálico aumentou a resistência final das próteses acrílicas provisórias. A interface de resina de conexão entre a barra de metal e os cilindros aumentou a resistência à carga de flexão.

\section{REFERÊNCIAS}

1 Van de Velde T, Collaert B, De Bruyn H. Immediate loading in the completely edentulous mandible: technical procedure and clinical results up to 3 years of functional loading. Clin Oral Implants Res. 2007 Jun;18(3):295-303. http://dx.doi.org/10.1111/j.16000501.2007.01340.x. PMid:17386064. [ Links ]

2 Maló P, Nobre MA, Lopes A, Moss SM, Molina GJ. A longitudinal study of the survival of All-on-4 implants in the mandible with up to 10 years of follow-up. J Am Dent Assoc. 2011 Mar;142(3):310-20. http://dx.doi.org/10.14219/jada.archive.2011.0170. PMid:21357865. [ Links ]

3 Maló P, Rangert B, Nobre M. “All-on-Four" immediate-function concept with Brånemark system ${ }^{\circledR}$ implants for completely edentulous mandibles: a retrospective clinical study. Clin Implant Dent Relat Res. 2003;5(Suppl 1):2-9. http://dx.doi.org/10.1111/j.17088208.2003.tb00010.x. PMid:12691645. [ Links ]

4 Correa S, Ivancik J, Isaza JF, Naranjo M. Evaluation of the structural behavior of three and four implant-supported fixed prosthetic restorations by finite element analysis. J Prosthodont Res. 2012 Apr;56(2):110-9. http://dx.doi.org/10.1016/j.jpor.2011.07.001. PMid:22104623. [ Links ]

5 Hinze $\mathrm{M}$, Thalmair T, Bolz W, Wachtel $\mathrm{H}$. Immediate loading of fixed provisional prostheses using four implants for the rehabilitation of the edentulous arch: a prospective clinical study. Int J Oral Maxillofac Implants. 2010 Sep-Oct;25(5):1011-8. PMid:20862417. [ Links ]

6 Begg T, Geerts GA, Gryzagoridis J. Stress patterns around distal angled implants in the allon-four concept configuration. Int J Oral Maxillofac Implants. 2009 Jul-Aug;24(4):663-71. PMid:19885406. [ Links ]

7 Ferreira EJ, Kuabara MR, Gulinelli JL. "All-on-four" concept and immediate loading for simultaneous rehabilitation of the atrophic maxilla and mandible with conventional and 
zygomatic implants. Br J Oral Maxillofac Surg. 2010 Apr;48(3):218-

20. http://dx.doi.org/10.1016/j.bjoms.2009.08.017. PMid:19767132. [ Links ]

8 Khatami AH, Smith CR. "All-on-Four" immediate function concept and clinical report of treatment of an edentulous mandible with a fixed complete denture and milled titanium framework. J Prosthodont. 2008 Jan;17(1):47-51. http://dx.doi.org/10.1111/j.1532849X.2007.00246.x. PMid:17931367. [ Links ]

9 Agliardi E, Panigatti S, Clericò M, Villa C, Maló P. Immediate rehabilitation of the edentulous jaws with full fixed prostheses supported by four implants: interim results of a single cohort prospective study. Clin Oral Implants Res. 2010 May;21(5):459-

65. http://dx.doi.org/10.1111/j.1600-0501.2009.01852.x. PMid:20105197. [ Links ]

10 Francetti L, Agliardi E, Testori T, Romeo D, Taschieri S, Del Fabbro M. Immediate rehabilitation of the mandible with fixed full prosthesis supported by axial and tilted implants: interim results of a single cohort prospective study. Clin Implant Dent Relat Res. 2008 Dec;10(4):255-63. http://dx.doi.org/10.1111/j.1708-8208.2008.00090.x.

PMid:18384405. [ Links ]

11 Ogawa T, Dhaliwal S, Naert I, Mine A, Kronstrom M, Sasaki K, et al. Impact of implant number, distribution and prosthesis material on loading on implants supporting fixed prostheses. J Oral Rehabil. 2010 Jul;37(7):525-31. http://dx.doi.org/10.1111/j.13652842.2010.02076.x. PMid:20236236. [ Links ]

12 Degidi M, Nardi D, Piattelli A. Prospective study with a 2-year follow-up on immediate implant loading in the edentulous mandible with a definitive restoration using intra-oral welding. Clin Oral Implants Res. 2010 Apr;21(4):379-85. http://dx.doi.org/10.1111/j.16000501.2009.01865.x. PMid:20128829. [ Links ]

13 Yilmaz B, Suarez C, McGlumphy E. Correction of misfit in a maxillary immediate metalresin implant-fixed complete prosthesis placed with flapless surgery on four implants. Int J Oral Maxillofac Implants. 2011 Sep-Oct;26(5):e23-8. PMid:22010097. [ Links ]

14 Borges AF, Pereira LAD, Thomé G, Melo AC, Sartori IAM. Prostheses removal for suture removal after immediate load: success of implants. Clin Implant Dent Relat Res. 2010 Sep;12(3):244-8. http://dx.doi.org/10.1111/j.1708-8208.2009.00157.x. PMid:19438945. [ Links ]

15 Engstrand P, Gröndahl K, Öhrnell LO, Nilsson P, Nannmark U, Branemark PI. Prospective follow-up study of 95 patients with edentulous mandibles treated according to the Brånemark Novum concept. Clin Implant Dent Relat Res. 2003;5(1):310. http://dx.doi.org/10.1111/j.1708-8208.2003.tb00176.x. PMid:12831723. [ Links ] 16 Landázuri-Del Barrio RA, Cosyn J, De Paula WN, De Bruyn H, Marcantonio E Jr. A prospective study on implants installed with flapless-guided surgery using the all-on-four 
concept in the mandible. Clin Oral Implants Res. 2013 Apr;24(4):428-

33. http://dx.doi.org/10.1111/j.1600-0501.2011.02344.x. PMid:22092825. [ Links ]

17 Inman DJ. Engineering vibrations. 3rd ed. Upper Saddle River: Prentice Hall; 2007. [ Links ] 18 Nikellis I, Levi A, Nicolopoulos C. Immediate loading of 190 endosseous dental implants: a prospective observational study of 40 patient treatments with up to 2-year data. Int J Oral Maxillofac Implants. 2004 Jan-Feb;19(1):116-23. PMid:14982364. [ Links ]

19 Wiskott HW, Nicholls JI, Belser UC. Stress fatigue: basic principles and prosthodontic implications. Int J Prosthodont. 1995 Mar-Apr;8(2):105-16. PMid:7575960. [ Links ]

20 Kim KS, Kim YL, Bae JM, Cho HW. Biomechanical comparison of axial and tilted implants for mandibular full-arch fixed prostheses. Int J Oral Maxillofac Implants. $2011 \mathrm{Sep}$ Oct;26(5):976-84. PMid:22010079. [ Links ]

21 Albakry M, Guazzato M, Swain MV. Biaxial flexural strength elastic moduli, and x-ray diffraction characterization of three prenssable all-ceramic materials. J Prosthet Dent. 2003 Apr;89(4):374-80. http://dx.doi.org/10.1067/mpr.2003.42. PMid:12690350. [ Links ]

22 Benazzi S, Kullmer O, Grosse IR, Weber GW. Using occlusal wear information and finite element analysis to investigate stress distributions in human molars. J Anat. 2011 Sep;219(3):259-72. http://dx.doi.org/10.1111/j.1469-7580.2011.01396.x. PMid:21615398. [ Links ]

23 Mericske-Stern R, Zarb GA. In vivo measurements of some functional aspects with mandibular fixed prostheses supported by implants. Clin Oral Implants Res. 1996 Jun;7(2):153-61. http://dx.doi.org/10.1034/j.1600-0501.1996.070209.x. PMid:9002834. [ Links ]

24 Brunski JB, Puleo DA, Nanci A. Biomaterials and biomechanics of oral and maxillofacial implants: current status and future developments. Int J Oral Maxillofac Implants. 2000 JanFeb;15(1):15-46. PMid:10697938. [ Links ]

25 Mostafa TM, Elgendy MN, Kashef NA, Halim MM. Evaluation of the precision of three implant transfer impression techniques using two elastomeric impression materials. Int J Prosthodont. 2010 Nov-Dec;23(6):525-8. PMid:21209987. [ Links ]

26 Drago CJ, Lazzara RJ. Immediate occlusal loading of Osseotite implants in mandibular edentulous patients: a prospective observational report with 18-month data. J Prosthodont. 2006 May-Jun;15(3):187-94. http://dx.doi.org/10.1111/j.1532-849X.2006.00099.x. PMid:16681501. [ Links ]

27- SILVA, Vanessa Araujo, et al. "Biomechanical development and evaluation of a new framework for all-on-four rehabilitation." Revista de Odontologia da UNESP 48 (2019). 
Desenvolvimento biomecânico e avaliação de uma nova estrutura para reabilitação all-on-four.

Vanessa Araujo SILVA

(C) (i)

This work is licensed under a Creative Commons Attribution 4.0 International

License. 